\title{
Clove Oil
}

National Cancer Institute

\section{Source}

National Cancer Institute. Clove Oil. NCI Thesaurus. Code C65346.

The essential oil extracted from Syzygium aromaticum. Clove oil has both analgesic and antiseptic properties and is commonly used to treat tooth and gum pain. 